Research Article

\title{
Bijections from Dyck and Motzkin meanders with catastrophes to pattern avoiding Dyck paths
}

\author{
Jean-Luc Baril*, Sergey Kirgizov \\ Laboratoire d'Informatique de Bourgogne, Université de Bourgogne Franche-Comté, France
}

(Received: 29 March 2021. Received in revised form: 26 April 2021. Accepted: 27 April 2021. Published online: 28 April 2021.)

(C) 2021 the authors. This is an open access article under the CC BY (International 4.0) license (www.creativecommons.org/licenses/by/4.0/).

\begin{abstract}
In this note, we present constructive bijections from Dyck and Motzkin meanders with catastrophes to Dyck paths avoiding some patterns. As a byproduct, we deduce correspondences from Dyck and Motzkin excursions to restricted Dyck paths.
\end{abstract}

Keywords: bijection; Dyck and Motzkin paths; meander and excursion with catastrophes; pattern avoidance.

2020 Mathematics Subject Classification: 05A15, 05A19.

\section{Introduction and notations}

The domain of lattice paths provides a very fertile ground for the combinatorial community. They have many applications in computer science, queuing theory, biology and physics [17], and there are a multitude of one-to-one correspondences with various combinatorial objects such as directed animals, pattern avoiding permutations, bargraphs, RNA structures and so on $[4,8,17]$. A recurring problem in combinatorics is the enumeration of these paths with respect to their length and other statistics [2,3,6,11-14,16]. In the literature, Dyck and Motzkin paths are the most often considered, possibly because they are, respectively, counted by the famous Catalan and Motzkin numbers (see A108 and A1006 in the Sloane's On-line Encyclopedia of Integer Sequences [15]).

Throughout this note, a lattice path is defined by a starting point $(0,0)$, an ending point $(n, k)$ with $n, k \geq 0$, it consists of steps lying in $S=\{(1, i): i \in \mathbb{Z}, i \leq 1\}$, and it never goes below the $x$-axis. The length of a path is the number of its steps. We denote by $\epsilon$ the empty path, i.e., the path of length zero. Constraining the steps to be in $\{(1,1),(1,-1)\}$ or $\{(1,1),(1,0),(1,-1)\}$, and fixing the end point on the $x$-axis, we retrieve the well-known definition of Dyck and Motzkin paths [17] respectively. Let $\mathcal{D}_{n}$ be the set of Dyck paths of semilength $n$, we define $\mathcal{D}=\cup_{n \geq 0} \mathcal{D}_{n}$. For short, we set $U=(1,1)$, $D=(1,-1), F=(1,0)$ and $D_{i}=(1,-i)$ for $i \geq 2$.

Considering these notations, a Motzkin meander with catastrophes is a lattice path where possible steps are $U, D, F$ and $D_{i}$ for $i \geq 2$, such that all steps $D_{i}$ end on the $x$-axis, and if we add the property that the path ends on the $x$-axis, we call it a Motzkin excursion with catastrophes (see [1]). Dyck meanders and Dyck excursions with catastrophes are those avoiding the step $F$. Let $\mathcal{M}_{n}$ (respectively $\mathcal{E}_{n}$ ) be the set of length $n$ Dyck meanders (respectively excursions) with catastrophes, and we set $\mathcal{M}=\cup_{n \geq 0} \mathcal{M}_{n}$ (respectively $\mathcal{E}=\cup_{n \geq 0} \mathcal{E}_{n}$ ). The sets of Motzkin meanders and excursions with catastrophes are respectively denoted by adding prime superscripts, $\mathcal{M}^{\prime}$ and $\mathcal{E}^{\prime}$. As mentioned in Corollary 2.4 in [1], the cardinality of $\mathcal{M}_{n}$ is given by the sequence A274115 in [15], and the cardinality of $\mathcal{E}_{n}$ is given by the sequence A224747. For instance, we have $U U D F U U F D_{3} U D U U U D D U D_{2} U U F F U F \in \mathcal{M}_{23}^{\prime}$ and $U U D U U D U D_{3} U D U U U D D U D_{2} \in \mathcal{E}_{17}$, and we refer to Figure 1 for an illustration of these two paths. Since Motzkin meanders with catastrophes can be obtained from Dyck meanders with catastrophes by possibly adding flat steps $F$, the ordinary generating function (o.g.f.) for the cardinality of $\mathcal{M}_{n}^{\prime}$ is given by

$$
\frac{M(x /(1-x))}{1-x}
$$

where

$$
M(x)=\frac{2 x}{2 x+(x+1)\left(\sqrt{1-4 x^{2}}-1\right)}
$$

is the o.g.f. for $\mathcal{M}_{n}$ (see [1]), which generates the $(n+1)$ th term of A54391. Simarly, $\mathcal{E}_{n}^{\prime}$ is counted by the $n$th term of A54391. 
(a)

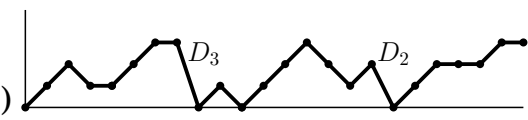

(b)

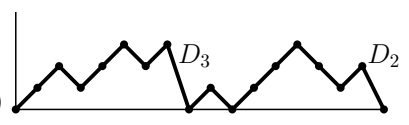

Figure 1: (a) A Motzkin meander with catastrophes in $\mathcal{M}_{23}^{\prime}$, and (b) a Dyck excursion with catastrophes in $\mathcal{E}_{17}$.

Dyck meanders with catastrophes was first introduced by Krinik et al. in [9] in the context of queuing theory. They correspond to the evolution of the queue by allowing some resets modeled by a catastrophe step $D_{i}$ for $i \geq 2$. Recently in [1], Banderier and Wallner provide many results about the enumeration and limit laws of these objects. Using algrebraic methods they prove that the set $\mathcal{M}_{n}$ of length $n$ Dyck meanders with catastrophes has the same cardinality as the set of equivalence classes of semilength $n+1$ Dyck paths modulo the positions of the pattern $D U U$, which in turn (see [10]) is in one-to-one correspondence with the set $\mathcal{A}_{n}$ of semilength $n$ Dyck paths avoiding occurrences at height $h>0$ of the patterns $U U U$ and $D U D$. They also provide a constructive bijection between $\mathcal{E}_{n}$ and the set of length $n$ Motzkin paths having their flat steps $F$ at height one.

The motivation of this work is to exhibit one-to-one correspondences between restricted Dyck paths (with no catastrophes) and the sets of paths with catastrophes $\mathcal{M}_{n}, \mathcal{E}_{n}, \mathcal{M}_{n}^{\prime}$, and $\mathcal{E}_{n}^{\prime}$. In Section 2 we present a constructive bijection between $\mathcal{M}_{n}$ and $\mathcal{A}_{n}$. Considering its restriction to excursions with catastrophes, we prove that $\mathcal{E}_{n}$ is in one-to-one correspondence with the set $\mathcal{A}_{n}^{\prime}$ of Dyck paths in $\mathcal{A}_{n}$ where any occurrence $U D$ on the $x$-axis appears before an occurrence of $U U U$ (not necessarily contiguous to the occurrence $U D$ ). This bijection establishes a curious correspondence connecting Dyck meanders with catastrophes and equivalence classes modulo the positions of $D U U$ in Dyck paths. In Section 3 we conduct the counterpart study for Motzkin meanders and excursions. More precisely, we exhibit a bijection between $\mathcal{M}_{n}^{\prime}$ and the set $\mathcal{B}_{n+1}$ of semilength $n+1$ Dyck paths avoiding the pattern $U U U$ at height $h \geq 2$, which also induces a bijection from the set $\mathcal{E}_{n}^{\prime}$ of Motzkin excursions with catastrophes to the set $\mathcal{B}_{n}$. The following table gives an overview of all these correspondences.

\begin{tabular}{r|ccc|l} 
Dyck meanders with cat. & $\mathcal{M}_{n}$ & $\rightarrow$ & $\mathcal{A}_{n}$ & Dyck paths avoiding $U U U$ and $D U D$ at $h>0$ \\
\hline Dyck excursions with cat. & $\mathcal{E}_{n}$ & $\rightarrow$ & $\mathcal{A}_{n}^{\prime}$ & $\mathcal{A}_{n}$ whose every $U D$ on the $x$-axis appears before $U U U$ \\
\hline Dyck paths & $\mathcal{D}_{n}$ & $\rightarrow$ & $\mathcal{A}_{2 n}^{\star}$ & Dyck paths starting with $U U$ and avoiding $U U U$ and $D U D$ \\
\hline Motzkin meanders with cat. & $\mathcal{M}_{n}^{\prime}$ & $\rightarrow$ & $\mathcal{B}_{n+1}$ & Dyck paths avoiding $U U U$ at $h \geq 2$ \\
\hline Motzkin excursions with cat. & $\mathcal{E}_{n}^{\prime}$ & $\rightarrow$ & $\mathcal{B}_{n}$ & \\
\hline Motzkin paths & $\mathcal{M}$ otz $_{n}$ & $\rightarrow$ & $\mathcal{B}_{n+1}^{\prime}$ & Dyck paths avoiding $U U U$ at $h \geq 2$ and $D U$ at $h=1$.
\end{tabular}

\section{Dyck meanders with catastrophes}

In this section we exhibit a constructive bijection between the set $\mathcal{M}_{n}$ of length $n$ Dyck meanders with catastrophes and the set $\mathcal{A}_{n}$ of semilength $n$ Dyck paths having no occurrence of the consecutive three steps $U U U$ and $D U D$ at height $h>0$ (or equivalently with a minimal ordinate $h>0$ ). We set $\mathcal{A}=\cup_{n \geq 0} \mathcal{A}_{n}$. Let us define recursively the map $\phi$ from $\mathcal{M}$ to $\mathcal{D}$ as follows. For $P \in \mathcal{M}$, we set

$$
\phi(P)=\left\{\begin{array}{llr}
\epsilon & \text { if } P=\epsilon, & (i) \\
U D \phi(\alpha) & \text { if } P=U \alpha, \\
U U D \phi(\alpha) D \phi(\beta) & \text { if } P=U \alpha D \beta, \\
U \phi(\alpha D) D \phi(\beta) & \text { if } P=U \alpha D_{2} \beta, \\
U D \phi\left(\alpha D_{i-1}\right) \phi(\beta) & \text { if } P=U \alpha D_{i} \beta \text { and } i \geq 3,
\end{array}\right.
$$

where $\beta \in \mathcal{M}$, and $\alpha$ is either the empty path or a lattice path consisting of $U$ - and $D$-steps such that $\alpha$ (respectively $\alpha D$, $\alpha D_{k-1}$ ) ends on the $x$-axis in the case (iii) (respectively $(i v),(v)$ ), and $\alpha$ does not necessarily end on the $x$-axis in the case (ii).

Due to the recursive definition, the image by $\phi$ of a length $n$ Dyck meander with catastrophes is a Dyck path of semilength $n$. For instance, the images of $U, U D, U U D_{2}, U U D U U D_{3}$ are respectively

We refer to Figure 2 for an illustration of this mapping. 


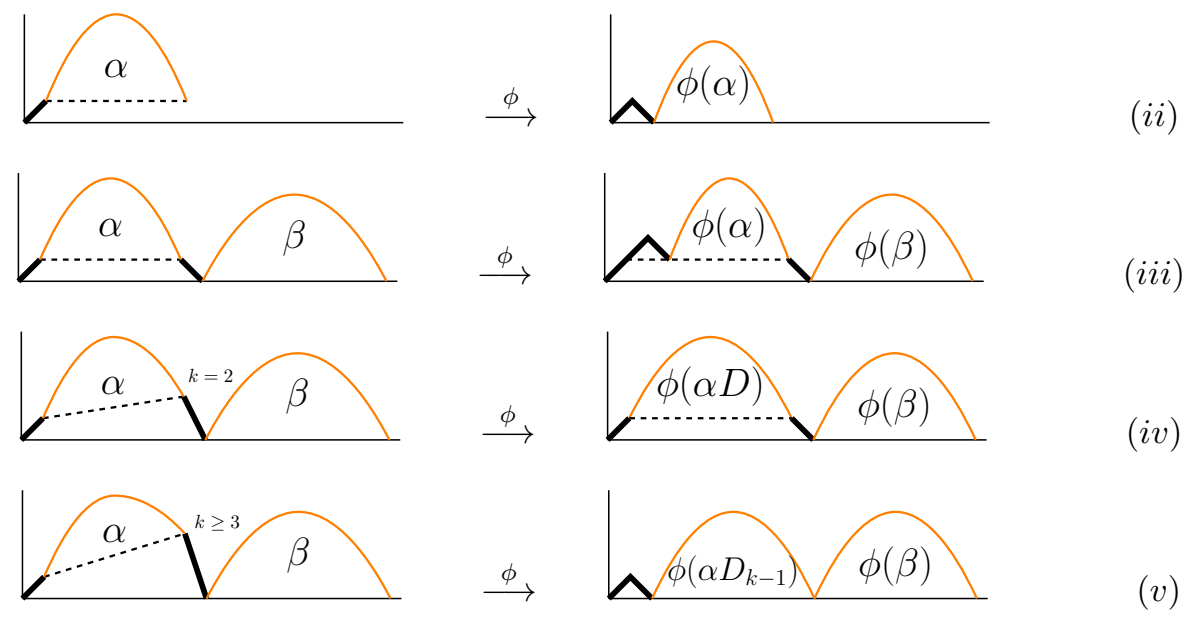

Figure 2: Illustration of the bijection $\phi$ between $\mathcal{M}_{n}$ and $\mathcal{A}_{n}$.

Lemma 2.1. For any $n \geq 0$,

- if $P \in \mathcal{M}_{n}$ then we have $\phi(P) \in \mathcal{A}_{n}$,

- if $P \in \mathcal{D}_{n}$ then we have $\phi(P) \in \mathcal{A}_{2 n}^{*}$, where $\mathcal{A}_{0}^{*}=\{\epsilon\}$ and for $n \geq 1$, the set $\mathcal{A}_{2 n}^{*}$ consists of semilength $2 n$ Dyck paths avoiding the patterns $U U U$ and DUD and starting with $U U D$.

Proof. We proceed by induction on $n$. The case $n=0$ is obvious. For $k \leq n$, we assume that for any $P \in \mathcal{D}_{k}$ we have $\phi(P) \in \mathcal{A}_{2 k}^{*}$ and for any $P \in \mathcal{M}_{k}$ we have $\phi(P) \in \mathcal{A}_{k}$. Now, let us prove the result for $k=n+1$.

Whenever $P \in \mathcal{D}_{n+1}$ we can write $P=U \alpha D \beta$ where $\alpha, \beta \in \mathcal{D}$. Thus, we have $\phi(P)=U U D \phi(\alpha) D \phi(\beta)$, and using the recurrence hypothesis on $\alpha$ and $\beta, \phi(P)$ is of semilength $2 n+2$, starts with $U U D$ and avoids the pattern $U U U$. Moreover $\phi(\alpha)$ (respectively $\phi(\beta)$ ) is either empty or it starts with $U U D$, which implies that $\phi(P)$ avoids $D U D$, and thus $\phi(P) \in \mathcal{A}_{2 n+2}^{*}$.

Now let us assume $P \in \mathcal{M}_{n+1}$.

- If $P=U \alpha$ with $\alpha \in \mathcal{M}_{n}$, then $\phi(P)=U D \phi(\alpha)$ and the recurrence hypothesis implies that $\phi(P)$ avoids $U U U$ and $D U D$ at height $h>0$.

- If $P=U \alpha D \beta$ where $\alpha \in \mathcal{D}$ and $\beta \in \mathcal{M}$, then the first part of the proof implies that $\phi(\alpha)$ avoids $U U U$ and $D U D$, and with the recurrence hypothesis on $\beta, \phi(P)=U U D \phi(\alpha) D \phi(\beta)$ belongs to $\mathcal{A}$.

- If $P=U \alpha D_{2} \beta$ and $\alpha D \in \mathcal{D}$, then using the first part of the proof $\phi(\alpha D)$ is not empty and avoids $U U U$ and $D U D$. The recurrence hypothesis implies that $\phi(P)=U \phi(\alpha D) D \phi(\beta)$ belongs to $\mathcal{A}$.

- If $P=U \alpha D_{i} \beta$ where $i \geq 3$ and $\alpha D_{i-1}$ ends on the $x$-axis, then using a simple induction on $i \geq 2, \phi\left(\alpha D_{i-1}\right)$ is not empty and avoids $U U U$ and $D U D$. The recurrence hypothesis implies that $\phi(P)=U \phi\left(\alpha D_{i-1}\right) D \phi(\beta) \in \mathcal{A}$.

The induction is completed.

Theorem 2.1. For $n \geq 0$, the map $\phi: \mathcal{M}_{n} \rightarrow \mathcal{A}_{n}$ is a bijection. Moreover, we have $\phi\left(\mathcal{D}_{n}\right)=\mathcal{A}_{2 n}^{*}$.

Proof. Due to the enumerative results in [1] (see Corollary 2.4) and the above lemma, it suffices to prove that $\phi$ is injective from $\mathcal{M}_{n}$ to $\mathcal{A}_{n}$. We proceed by induction on $n$. The case $n=0$ is obvious. For $k \leq n$, we assume that $\phi$ is an injection from $\mathcal{M}_{k}$ to $\mathcal{A}_{k}$, and we prove the result for $k=n+1$.

According to the definition of $\phi$ and Lemma 2.1, the image by $\phi$ of $P \in \mathcal{M}$ satisfying (ii) is a Dyck path starting by $(U D)^{k} R$ for some $k \geq 1$ where $R$ is a Dyck path in $\mathcal{A}_{2 i}^{*}$ for some $i \geq 0$, which means that $R$ avoids $U U U$; a meander satisfying (iii) is sent by $\phi$ to a Dyck path in $\mathcal{A}_{2 i}^{*}$ for some $i \geq 1$; a meander satisfying (iv) is sent to a Dyck path starting with $U U U D$; and a meander satisfying $(v)$ is sent to a Dyck path starting with $(U D)^{k}$ for some $k \geq 1$ and such that it contains an occurrence $U U U$ on the $x$-axis. Then, for $P, Q \in \mathcal{M}_{n+1}, \phi(P)=\phi(Q)$ implies that $P$ and $Q$ belong to the same case (i), (ii), (iii), (iv) or $(v)$. So, the recurrence hypothesis induces $P=Q$ which completes the induction. Thus $\phi$ is injective. Since $\mathcal{M}_{n}$ and $\mathcal{A}_{n}$ have the same cardinality (see [1] and A274115 in [15]), $\phi$ is a bijection.

Considering the previous lemma, it suffices to check that $\mathcal{A}_{2 n}^{*}$ is counted by the Catalan numbers in order to prove $\phi\left(\mathcal{D}_{n}\right)=\mathcal{A}_{2 n}^{*}$. A Dyck path $P \in \mathcal{A}_{2 n}^{*}$ is either empty or it consists of a sequence of $U U D \alpha D$ where $\alpha$ belongs to $\mathcal{A}_{2 n-2}^{*}$. 
Let $A^{*}(x)$ be the generating function for the cardinality of $\mathcal{A}_{2 n}^{*}$ (with respect to the semilength). We obtain the following functional equations

$$
A^{*}(x)=1+\frac{x^{2} A^{*}(x)}{1-x^{2} A^{*}(x)}
$$

which implies that $\mathcal{A}_{2 n}^{*}$ is counted by the $n$th Catalan number. Therefore $\phi: \mathcal{D}_{n} \rightarrow \mathcal{A}_{2 n}^{*}$ is a bijection.

Remark 2.1. In [10], it is proven that the set $\mathcal{A}_{n}$ is a representative set of the equivalence classes modulo the pattern DUU on Dyck paths, i.e. two Dyck paths $P$ and $Q$ are equivalent if and only if the positions of the occurrences DUU are the same in $P$ and $Q$ (see also [3]). So, the bijection $\phi$ establishes a direct correspondence between these classes and Dyck meanders with catastrophes.

Let $\mathcal{A}_{n}^{\prime}$ be the subset of $\mathcal{A}_{n}$ consisting of paths $P$ such that any occurrence $U D$ on the $x$-axis in $P$ appears before an occurrence of $U U U$ (not necessarily contiguous to the occurrence $U D$ ). The next theorem gives a bijection between $\mathcal{A}_{n}^{\prime}$ and the set $\mathcal{E}_{n}$ of length $n$ Dyck excursions with catastrophes.

Theorem 2.2. For $n \geq 0$, we have $\phi\left(\mathcal{E}_{n}\right)=\mathcal{A}_{n}^{\prime}$.

Proof. Thanks to Theorem 2.1, it suffices to check that for any $P \in \mathcal{E}_{n}, \phi(P) \in \mathcal{A}_{n}^{\prime}$, and $\left|\mathcal{A}_{n}^{\prime}\right|=\left|\mathcal{E}_{n}\right|$. Any $P \in \mathcal{E}_{n}$ satisfies one of the cases $(i),(i i i),(i v)$ and $(v)$ with $\beta \in \mathcal{E}$. We proceed by induction on the length in order to prove that $\phi(P) \in \mathcal{A}_{n}^{\prime}$. The case $(i)$ is obvious. Whenever $P$ satisfies the cases $(i i i)$ or $(i v)$, the only possibility for an occurrence of $U D$ to appear at height zero in $\phi(P)=U U D \phi(\alpha) D \phi(\beta)$ (respectively $\phi(P)=U \phi(\alpha D) D \phi(\beta)$ ) is to be inside $\phi(\beta)$. Applying the recurrence hypothesis on $\beta, \phi(P) \in \mathcal{A}_{n}^{\prime}$. For a path $P$ satisfying the case $(v)$, we have seen in the proof of Theorem 2.1 that $\phi(P)$ starts necessarily with $(U D)^{k}$ for $k \geq 1$ followed by $U U U$. Using the recurrence hypothesis for $\beta$, we obtain $\phi(P) \in \mathcal{A}_{n}^{\prime}$. The induction is completed.

Now, let us prove that $\left|\mathcal{A}_{n}^{\prime}\right|=\left|\mathcal{E}_{n}\right|$. Any path $P \in \mathcal{A}_{n}^{\prime}$ satisfies one of the following two cases: (a) $P \in \mathcal{A}_{n}$ does not contain any occurrence $U D$ on the $x$-axis, and (b) $P=Q U D R$ where $Q \in \mathcal{A}$ and $R \in \mathcal{A}$ such that $R$ contains at least one occurrence of $U U U$ and avoids any occurrence $U D$ on the $x$-axis. Let $\mathcal{K}$ (respectively $\overline{\mathcal{K}}$ ) be the set of Dyck paths in $\mathcal{A}$ satisfying $(a)$ (respectively (b)), and let $K(x)$ and $\bar{K}(x)$ be the corresponding generating functions for their cardinalities with respect to the semilength. Obviously, the generating function $A^{\prime}(x)$ for $\mathcal{A}^{\prime}=\cup_{n \geq 0} \mathcal{A}_{n}$ satisfies

$$
A^{\prime}(x)=K(x)+\bar{K}(x) .
$$

A nonempty path $P \in \mathcal{K}$ can be decomposed $P=U \alpha D \beta$ where $\beta \in \mathcal{K}$ and $\alpha$ is a nonempty Dyck path avoiding $U U U$ and $D U D$. Then either $\alpha \in \mathcal{A}^{\star} \backslash\{\epsilon\}$ or $\alpha=U D \alpha^{\prime}$ with $\alpha^{\prime} \in \mathcal{A}^{\star}$. Thus the generating function for $\mathcal{K}$ is given by

$$
K(x)=1+x\left(A^{\star}(x)-1+x A^{\star}(x)\right) \cdot K(x) .
$$

Due to the form of a path $P \in \overline{\mathcal{K}}$, we deduce the functional equation

$$
\bar{K}(x)=A(x) x R(x)
$$

where $A(x)$ is the generating function for $\mathcal{A}$ and $R(x)$ is the generating function for the paths in $\mathcal{A}$ avoiding any occurrence $U D$ on the $x$-axis and containing at least one occurrence of $U U U$. Due to Theorem 2.1, $A(x)$ is also the o.g.f. for Dyck meanders with catastrophes that is

$$
A(x)=M(x)=\frac{2 x}{2 x+(x+1)\left(\sqrt{1-4 x^{2}}-1\right)} .
$$

Then, we have $R(x)=K(x)-L(x)$ where $L(x)$ is the generating function for the set $\mathcal{L}$ of Dyck paths in $\mathcal{A}$ avoiding $U D$ and $U U U$ on the $x$-axis. Note that $\mathcal{L}$ is exactly the set $\mathcal{A}^{\star}$, then

$$
R(x)=K(x)-A^{\star}(x) .
$$

Combining the previous equations, we obtain

$$
A^{\prime}(x)=\frac{2-3 x-2 x^{2}+x \sqrt{1-4 x^{2}}}{2-2 x-4 x^{2}-2 x^{3}}
$$

which is exactly the generating function of $\mathcal{E}_{n}$ found by [1]. 


\section{Motzkin meanders with catastrophes}

In this section we exhibit a constructive bijection between the set $\mathcal{M}_{n}^{\prime}$ of length $n$ Motzkin meanders with catastrophes and the set $\mathcal{B}_{n+1}$ of semilength $n+1$ Dyck paths avoiding the patterns $U U U$ at height $h \geq 2$. Before defining this bijection we recall that there exists a one-to-one correspondence $\chi$ between length $n$ Motzkin paths and semilength $n$ Dyck paths avoiding $U U U$. From a Dyck path avoiding $U U U$, we replace each $U U D$ with $U$, and we replace each remaining $U D$ with $F$. For instance, the image by $\chi$ of $U F U D D$ is $U U D U D U U D D D$ (see $[5,7]$ ).

Now, let us use $\chi$ in order to define recursively the map $\psi$ from $\mathcal{M}^{\prime}$ to $\mathcal{D}$ as follows. For $P \in \mathcal{M}^{\prime}$, we set

$$
\psi(P)= \begin{cases}U D & \text { if } P=\epsilon \\ U D \psi(\alpha) & \text { if } P=F \alpha \\ U U \chi\left(\alpha_{1}\right) D U \chi\left(\alpha_{2}\right) D \ldots U \chi\left(\alpha_{k}\right) D D & \text { if } P=U \alpha_{1} U \alpha_{2} \ldots U \alpha_{k} \\ U U \chi\left(\alpha_{1}\right) D U \chi\left(\alpha_{2}\right) D \ldots U \chi\left(\alpha_{k}\right) D D \psi(\beta) & \text { if } P=U \alpha_{1} U \alpha_{2} \ldots U \alpha_{k} D_{k} \beta\end{cases}
$$

where $k \geq 1, \alpha, \beta \in \mathcal{M}^{\prime}$, and $\alpha_{1}, \alpha_{2}, \ldots, \alpha_{k}$ are some possibly empty Motzkin paths (considering that $D_{1}$ is defined to be $D$ ).

Clearly, the image by $\psi$ of a length $n$ Motzkin meander with catastrophes is a Dyck path of semilength $n+1$. For instance, the images by $\psi$ of $\epsilon, F, U D, U U D_{2}, U U D U U D_{3}$ are respectively

\section{$U D, U D U D, U U D D U D, U U D U D D U D, U U U U D D U D U D D U D$.}

We refer to Figure 3 for an illustration of this mapping.
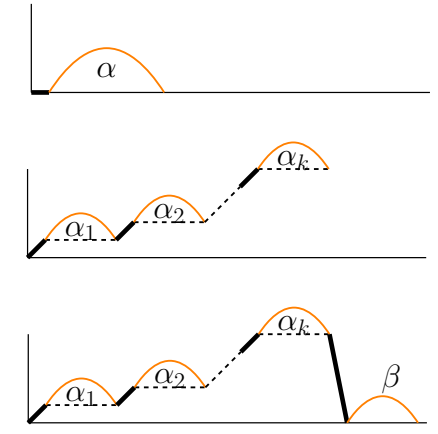
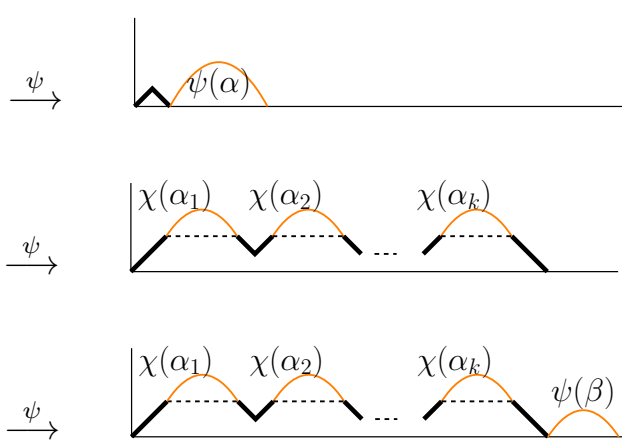

(ii)

Figure 3: Illustration of the bijection $\psi$ between $\mathcal{M}_{n}^{\prime}$ and $\mathcal{B}_{n+1}$.

A simple observation provides the following results.

Theorem 3.1. For $n \geq 0$, the map $\psi$, defined above, induces a bijection from $\mathcal{M}_{n}^{\prime}$ to $\mathcal{B}_{n+1}$. Moreover, the image of the set of length $n$ Motzkin paths is the set of semilength $n+1$ Dyck paths avoiding UUU at height $h \geq 2$ and the pattern DU at height one.

Corollary 3.1. For $n \geq 0, \psi\left(\mathcal{E}_{n}^{\prime}\right)$ is the set of Dyck paths in $\mathcal{B}_{n+1}$ ending with $U D$, which implies that $\psi$ induces a one-to-one correspondence from paths $P \in \mathcal{E}_{n}^{\prime}$ to $\mathcal{B}_{n}$ after deleting the last two steps $U D$ from $\psi(P)$.

\section{Acknowledgment}

We would like to thank Cyril Banderier for suggesting us to explore constructive bijections between meanders with catastrophes and Dyck paths avoiding some patterns.

\section{References}

[1] C. Banderier, M. Wallner, Lattice paths with catastrophes, Discrete Math. Theor. Comput. Sci. 19 (2017) Art\# 23.

[2] E. Barcucci, A. Del Lungo, S. Fezzi, R. Pinzani, Nondecreasing Dyck paths and q-Fibonacci numbers, Discrete Math. 170 (1997) $211-217$.

[3] J.-L. Baril, A. Petrossian, Equivalence classes of Dyck paths modulo some statistics, Discrete Math. 338 (2015) 655-660.

[4] J.-L. Baril, D. Bevan, S. Kirgizov, Bijections between directed animals, multisets and Grand-Dyck paths, Electron. J. Combin. 27 (2020) Art\# P2.10.

[5] D. Callan, Two bijections for Dyck path parameters, arXiv:math/0406381v2 [math.CO], (2004).

[6] E. Deutsch, Dyck path enumeration, Discrete Math. 204 (1999) 167-202.

[7] S. Elizalde, T. Mansour, Restricted Motzkin permutations, Motzkin paths, continued fractions, and Chebyshev polynomials, Discrete Math. 305 (2005) $170-189$.

[8] D. E. Knuth, The Art of Computer Programming, Volume 1. Fundamental Algorithms, Addison-Wesley, Reading, 1973.

[9] A. Krinik, G. Rubino, D. Marcus, R. J. Swift, H. Kasfy, H. Lam, Dual processes to solve single server systems, J. Statist. Plann. Inference 135 (2005) 121-147. 
[10] K. Manes, A. Sapounakis, I. Tasoulas, P. Tsikouras, Equivalence classes of ballot paths modulo strings of length 2 and 3, Discrete Math. 339 (2016) $2557-2572$

[11] T. Mansour, Statistics on Dyck paths, J. Integer Seq. 9 (2006) Art\# 06.1.5.

[12] D. Merlini, R. Sprugnoli, M. C. Verri, Some statistics on Dyck paths, J. Statist. Plann. Inference 101 (2002) $211-227$.

[13] A. Panayotopoulos, A. Sapounakis, On the prime decomposition of Dyck paths, J. Combin. Math. Combin. Comput. 40 (2002) 33-39.

[14] A. Sapounakis, I. Tasoulas, P. Tsikouras, Counting strings in Dyck paths, Discrete Math. 307 (2007) 2909-2924.

[15] N. J. A. Sloane, The On-line Encyclopedia of Integer Sequences, http://oeis . org

[16] Y. Sun, The statistic "number of udu's" in Dyck paths, Discrete Math. 287 (2004) 177-186.

[17] R. P. Stanley, Enumerative Combinatorics, Volume 2, Cambridge University Press, Cambridge, 1999. 